\title{
Análisis de la mortalidad semanal como herramienta para la detección temprana de alertas en salud pública
}

\author{
Analysis of weekly mortality as a tool for the early detection of alerts \\ in public health
}

\author{
J. Etxeberria ${ }^{1,2}$, Y. Floristán ${ }^{1,2}$, E. Ardanaz ${ }^{1,2}$, R. López-Escudero ${ }^{1,2}$, J. Castilla ${ }^{1,2}$
}

\section{RESUMEN}

Fundamento. En salud pública tiene interés creciente monitorizar indicadores en tiempo real que posibiliten la actuación inmediata. Se plantea como objetivo del estudio evaluar la utilidad del análisis de la mortalidad semanal para la detección temprana de alertas en salud pública.

Métodos. Se analizó la mortalidad de recepción temprana (MRT) que proviene de cinco registros civiles informatizados de Navarra y recogen el $70 \%$ de la mortalidad total (MT) para el periodo 2003-2007. Se identificaron las semanas en las que la mortalidad registrada había sobrepasado el umbral calculado a partir de los 5 años precedentes. Se analizó la correspondencia de estos excesos de mortalidad con los niveles de incidencia de gripe y con las temperaturas ambientales elevadas.

Resultados. La concordancia entre las alertas detectadas a partir de la información de las fuentes de MRT y MT fue moderada (índice Kappa 0,59; IC 95\% 0,42-0,76). Ambas fuentes se comportaron de forma análoga en la detección de excesos de mortalidad cuando estos ocurrieron en semanas con actividad gripal, con 13 y 12 alertas respectivamente. En relación a las temperaturas elevadas, la MT generó alertas únicamente en los años en los que se declararon olas de calor, mientras que la MRT también las generó en otras semanas calurosas, pero que no alcanzaron el umbral de ola de calor.

Conclusión. La disponibilidad temprana de resultados, la concordancia aceptable entre fuentes y el alto porcentaje de excesos de mortalidad que pueden atribuirse a problemas de salud pública identificados, muestran la potencial utilidad de la monitorización de la mortalidad semanal en vigilancia epidemiológica.

Palabras clave. Vigilancia epidemiológica. Vigilancia sindrómica. Mortalidad. Alerta temprana. Gripe. Ola de Calor

\begin{abstract}
Background. There is a growing interest in public health in monitoring indicators in real time that permit immediate action to be taken. The aim of this study is to evaluate the utility of the analysis of weekly mortality for the early detection of alerts in public health.
\end{abstract}

Methods. We analysed the early reception of mortality (ERM) from five computerised civil registers in Navarre and gathered $70 \%$ of total mortality (TM) for the 20032007 period. We identified the weeks when mortality exceeded the threshold calculated on the basis of the five previous years. We analysed the correspondence of these excesses in mortality with flu levels of incidence and with high atmospheric temperatures.

Results. The was moderate concordance between the alerts detected from the information proceeding from the ERM and TM sources (Kappa index 0.59; CI 95\% $0.42-0.76)$. Both sources behaved analogously in the detection of excesses of mortality when these occurred in weeks of severe flu, with 13 and 12 alerts respectively. In relation to high temperatures, TM only generated alerts in the years when heat waves were declared, while ERM also generated them in other weeks which were hot but did not reach the heat wave threshold.

Conclusion. The early availability of results, the acceptable concordance between sources and the high percentage of excesses of mortality attributable to identified public health problems, show the potential utility of monitoring weekly mortality in epidemiological surveillance.

Key words. Epidemiological surveillance. Syndromic surveillance. Mortality. Early alert. Flu. Heat wave.
1. Instituto de Salud Pública de Navarra.

2. Ciber de Epidemiología y Salud Pública (CIBERESP).

Recepción el 17 de marzo de 2009

Aceptación provisional el 30 de diciembre de 2009

Aceptación definitiva el 8 de marzo de 2010

Ayudas obtenidas para este trabajo

FIS PI 06/1346 y CIBER Epidemiología y Salud Pública

\section{Correspondencia}

Jaione Etxeberria Andueza

Instituto de Salud Pública de Navarra

Leyre, 15

31003 Pamplona

E-mail: jaione.etxeberria.andueza@cfnavarra.es 


\section{INTRODUCCIÓN}

En salud pública tiene interés creciente monitorizar indicadores de salud a tiempo real que posibiliten la actuación de forma temprana. El análisis de la mortalidad es una de las herramientas básicas en vigilancia de salud pública. La relación entre las temperaturas ambientales y mortalidad se viene estudiando desde años atrás. Se pueden encontrar en la bibliografía referencias donde se concluye que tanto las bajas temperaturas como las extremadamente altas se relacionan con el incremento de la mortalidad $^{1-5}$. En invierno, la relación entre bajas temperaturas y mortalidad se asocia principalmente a enfermedades cardiovasculares y causas respiratorias y la circulación del virus de la gripe parece jugar un papel importante ${ }^{3}$.

Pero es la mortalidad durante el verano la que ha recibido mayor atención debido a las altas temperaturas. La ola de calor registrada en Europa en el año 2003 ${ }^{2}$ y la repercusión que tuvo sobre la mortalidad, hizo que los organismos de salud pública reforzaran la monitorización de indicadores de salud a tiempo real para detectar situaciones de emergencia sanitaria antes de lo que sería posible con los métodos tradicionales de salud pública y así poder ayudar a las autoridades sanitarias en una rápida intervención. El Centro Nacional de Epidemiología desarrolla desde el año 2004 el análisis de la mortalidad relacionada con los cambios de temperatura ambiental ${ }^{3}$. Ese mismo año, en $\mathrm{Na}$ varra también se puso en marcha el Plan de Prevención de la ola de calor con el objetivo principal de disminuir la morbimortalidad ocasionada por el aumento de la temperatura ambiental y con objetivos más específicos de informar a la población y a los profesionales del riesgo asociado a aumentos de temperatura y situaciones de calor extremo ${ }^{6}$.

Desde esta perspectiva, se plantea como objetivo de este trabajo estudiar la posible utilidad del análisis de la mortalidad semanal como herramienta para la vigilancia epidemiológica. Para ello, se estudia la correspondencia entre los excesos de mortalidad con los históricos de gripe y temperaturas ambientales en el quinquenio 2003-2007 y la concordancia entre los análisis realizados con fuentes de mortalidad disponibles precozmente y el registro de mortalidad completo.

\section{MATERIAL Y MÉTODOS}

\section{Fuentes de información de mortalidad}

Entre las fuentes de mortalidad disponibles en Navarra, se han seleccionado dos para llevar a cabo este estudio. La mortalidad de recepción temprana (MRT) proviene de cinco registros civiles informatizados de Navarra correspondientes a Pamplona, Tudela, Tafalla, Estella y Aoiz recogidos por el Ministerio de Justicia. Está disponible con un máximo de 48 horas de retraso y corresponde aproximadamente al $70 \%$ de la mortalidad total. La fuente MRT puede tener oscilaciones en el periodo de una semana debido al retraso administrativo, pudiéndose considerar completa en el transcurso de dicho periodo. La segunda fuente, la mortalidad total (MT) incluye el total de fallecidos en Navarra y está disponible a más largo plazo. Tanto MRT como MT originalmente se disponen en registros individualizados por personas y para el presente análisis utilizamos únicamente la variable fecha de defunción.

\section{Fuentes de información de gripe y temperaturas}

La información de gripe se obtuvo del sistema de enfermedades de declaración obligatoria (EDO) y de la Red de médicos centinela de atención primaria para la vigilancia de la gripe ${ }^{7}$. La información relacionada con la temperatura fue obtenida de la web de meteorología y climatología de Navarra ${ }^{8}$. En esta web se dispone, entre otras cosas, de la temperatura máxima y mínima diaria de la estación meteorológica de Noáin. Se selecciona esta estación por ser propiedad de la Agencia Estatal de Meteorología, por ser una estación automatizada y disponer de los datos histó- 
ricos necesarios para llevar a cabo este estudio. Se consideraron las temperaturas diarias de máximos y mínimos desde el $01 / 01 / 2003$ hasta $31 / 12 / 2007$, completando los datos no disponibles con el promedio de las temperaturas de los tres días anteriores.

Toda la información recogida de las fuentes anteriores (tanto de mortalidad, como de gripe y temperaturas) fue analizada por semanas epidemiológicas y años epidemiológicos para el quinquenio 2003-2007. La semana epidemiológica es la estandarización del tiempo que se establece para fines de vigilancia que permite la comparación de eventos sucedidos en determinado año o período dentro de un año con años anteriores. Las semanas inician en domingo a las 00:00 h y terminan en sábado a las 24:00 h. Para designar la primera semana epidemiológica del año, se ubica el primer sábado de enero que incluya en los días inmediatamente precedentes, cuatro o más días del mes de enero, aún cuando esa primera semana se inicie en diciembre.

Tanto en las fuentes de mortalidad como de gripe, las observaciones consideradas para cada semana corresponden a la suma de los casos observados en los días que incluye cada una de las semanas. En relación a las temperaturas, para cada semana epidemiológica se consideraron dos observaciones: la media de las máximas y la media de las mínimas de las temperaturas observadas en dicha semana.

\section{Definición de los umbrales de mortalidad}

Para evaluar los excesos de mortalidad en las fuentes MRT y MT se definieron los llamados umbrales de mortalidad. La técnica de los umbrales es una metodología utilizada y validada ${ }^{9}$ y permite evaluar excesos en la mortalidad para la razón $\left(\mathrm{R}_{\mathrm{i}}\right)$ entre casos observados de mortalidad respecto a los casos esperados en una semana epidemiológica concreta:

$\mathrm{R}_{\mathrm{i}}=$ casos observados en la semana i / casos esperados en la semana $i$
Para la definición de los casos esperados en una semana se consideró la información histórica. Concretamente, para cada semana, los casos esperados se obtuvieron como la media de las muertes registradas en 3 semanas (la misma, la anterior y la posterior) de los cinco años anteriores (media histórica).

Así, el cálculo del umbral de mortalidad para la semana i se calculó de la siguiente forma: $U_{i}=1+1,96\left(\operatorname{SD}_{i} / \bar{X}_{i}\right)$, siendo $\bar{X}_{i}$ la media histórica y $\mathrm{SD}_{\mathrm{i}}$ la desviación estándar de los 15 datos considerados. En este análisis se ha tolerado un error del $5 \%$. Cuando el valor Ri supera el valor Ui se considera un exceso de mortalidad en dicha semana y se genera una alerta.

Los umbrales de mortalidad se calcularon para el periodo 2003-2007 y de forma independiente para MRT y MT por lo que se consideraron los individuos con fecha de defunción comprendidos en el rango 01/01/1998-31/12/2007. Se eliminaron los individuos fallecidos en la semana 53 de 2003 para unificar el número de semanas epidemiológicas anuales.

Con las alertas generadas por cada una de las fuentes de mortalidad se realizó un análisis cuantitativo. Se computaron las alertas generadas por MRT y MT coincidiendo en el periodo de \pm 1 semana considerando que las alertas pueden verse desplazadas dependiendo de las fuentes. Para estudiar la concordancia de las alertas se utilizó el índice Kappa de concordancia y su correspondiente intervalo de confianza ${ }^{10}$. A continuación, se estudió la utilidad de MRT y MT como herramienta para la vigilancia evaluando la correspondencia entre los excesos de mortalidad con la evolución de la circulación del virus gripal y las medias de los máximos y mínimos de temperaturas observadas en cada una de las semanas. Se considera temporada gripal cuando el número de casos de gripe supera el umbral epidemiológico establecido en 68 casos por $100.000^{7}$ y se define ola de calorépoca de calor para Navarra cuando la temperatura mínima este por encima de $21,5^{\circ}$ y la máxima supere los $35,8^{\mathrm{o} 6}$. 


\section{RESULTADOS}

\section{Comparación de las fuentes MRT y MT en la detección de los excesos de mortalidad}

En este primer punto se muestran los resultados del análisis cuantitativo de las alertas generadas por las fuentes MRT y MT así como las representaciones gráficas de los umbrales para los años comprendidos en el quinquenio 2003-2007. En la tabla 1 se muestra el análisis descriptivo sobre el número de muertes observado en cada uno de los años.

Tabla 1. Análisis descriptivo del número de muertes semanales según las fuentes de mortalidad de recepción temprana (MRT) y de mortalidad total (MT) en el quinquenio 2003-2007.

\begin{tabular}{lccccc}
\hline & $\mathbf{2 0 0 3}$ & $\mathbf{2 0 0 4}$ & $\mathbf{2 0 0 5}$ & $\mathbf{2 0 0 6}$ & $\mathbf{2 0 0 7}$ \\
\hline Mortalidad de recepción temprana & & & & & \\
\hline Mínimo & 43 & 42 & 49 & 51 & 41 \\
Máximo & 89 & 88 & 102 & 88 & 98 \\
Media & 65 & 62 & 70 & 66 & 70 \\
Mediana & 65 & 63 & 70 & 67 & 71 \\
Rango intercuartílico & 12 & 14 & 16 & 14 & 15 \\
\hline Mortalidad total & & & & & \\
\hline Mínimo & 68 & 66 & 71 & 77 & 68 \\
Máximo & 139 & 123 & 161 & 121 & 132 \\
Media & 102 & 95 & 100 & 95 & 99 \\
Mediana & 102 & 98 & 97 & 94 & 101 \\
Rango intercuartílico & 12 & 16 & 14 & 12 & 18 \\
\hline
\end{tabular}

La figura 1 muestra la representación gráfica de los umbrales de mortalidad para MRT (columna de la izquierda) y MT (columna de la derecha). En cada uno de los gráficos, se distinguen de color rojo los umbrales de mortalidad y de color azul la razón entre los casos observados y esperados. Cuando la línea de color azul supera la línea roja, se considera que un exceso de mortalidad. A la vista de las representaciones obtenidas, son apreciables las discrepancias entre las alertas detectadas para cada fuente de mortalidad. Exceptuando la representación de los años 2003 y 2006, las alertas generadas por cada una de las fuentes parecen tener un comportamiento distinto. 


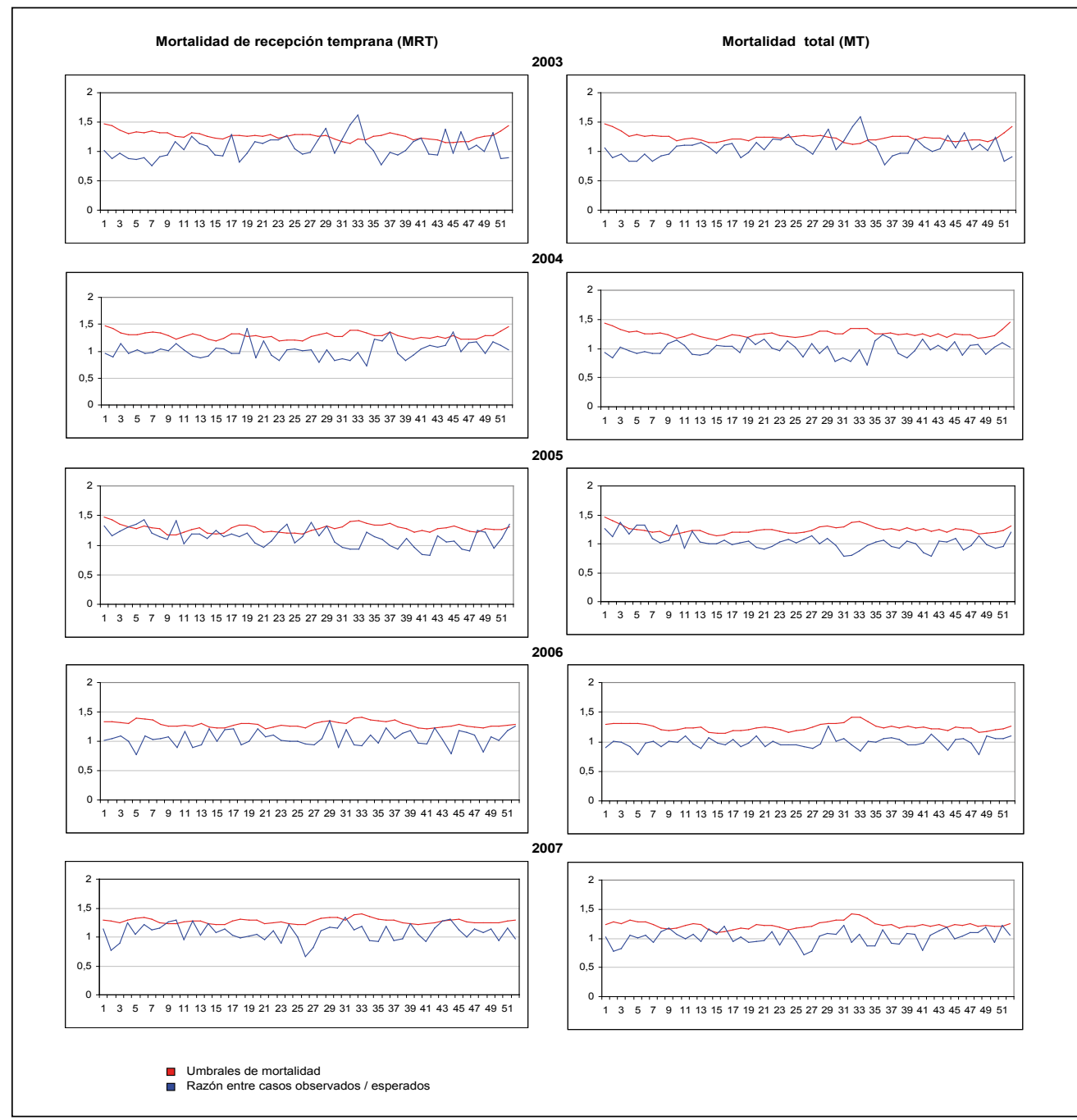

Figura 1. Umbrales y razones de mortalidad generados por la fuente de mortalidad de recepción temprana MRT (columna de la izquierda) y mortalidad total MT (columna de la derecha). De color rojo se muestran los umbrales de mortalidad y de color azul la razón entre los casos observados y esperados. Cuando la línea de color azul supera la línea roja, se considera que un exceso de mortalidad (alerta).

Para cuantificar dichas diferencias, en la tabla 2 se muestran el número de alertas generadas por MRT y MT. En las dos primeras filas se computa el número de alertas generadas en cada año y en el total de los cinco años considerados. En la tercera fila se muestran las alertas coincidentes (Tabla 2, fila 3) y no coincidentes (Tabla 2, fila 4) en el rango de \pm 1 semana. En el cómputo total de los cinco años estudiados el número de veces que se supera el umbral con MRT es mayor que MT, 29 frente a 19: 10 y 9 para 2003, 3 y 1 para 2004, 11 y 4 para 2005, 0 y 0 para 2006 y 5 y 5 para 2007 respectivamente. Tal y como se aprecia en estos datos, la mayor diferencia en el número 
de alertas se observó en el año 2005. Las alertas coincidentes en el periodo de \pm 1 semana son: 9, 1, 3, 2, en 2003, 2004, 2005 y 2007 respectivamente. En 2006 ninguna de las fuentes genera alertas. En el quinquenio completo 2003-2007, el número de alertas coincidentes son 15 (79,0\% de las alertas generadas por MT).

Tabla 2. Número de alertas generadas por las fuentes de mortalidad de recepción temprana (MRT) y mortalidad total (MT) para el quinquenio 2003-2007.

\begin{tabular}{lcccccc}
\hline & $\mathbf{2 0 0 3}$ & $\mathbf{2 0 0 4}$ & $\mathbf{2 0 0 5}$ & $\mathbf{2 0 0 6}$ & $\mathbf{2 0 0 7}$ & Total \\
\hline Mortalidad de recepción temprana & 10 & 3 & 11 & 0 & 5 & $\mathbf{2 9}$ \\
\hline Mortalidad Total & 9 & 1 & 4 & 0 & 5 & $\mathbf{1 9}$ \\
\hline Alertas de MRT y MT coincidentes en \pm 1 semana & 9 & 1 & 3 & 0 & 2 & $\mathbf{1 5}$ \\
Alertas generadas por MRT no coincidentes con MT & 1 & 2 & 8 & 0 & 3 & $\mathbf{1 4}$ \\
Alertas generadas por MT no coincidentes con MRT & 0 & 0 & 1 & 0 & 3 & $\mathbf{4}$ \\
\hline
\end{tabular}

Para estudiar la concordancia de MRT y MT se consideró también el índice Kappa y su intervalo se confianza. En la tabla 3 se resumen el número de alertas coincidentes y discordantes de las fuentes para las 260 semanas (52 en cada uno de los cinco años considerados). El índice Kappa obtenido para esta tabla es de $0,59(\mathrm{p}<0,0001)$, con un intervalo de confianza al $95 \%$ de 0,42 0,76 . Con este índice Kappa se concluye una concordancia moderada, considerando concordancia moderada un valor comprendido entre 0,41 y 0,60 .

Tabla 3. Concordancia entre de alertas semanales generadas por la mortalidad de recepción temprana y las generadas por mortalidad total.

\begin{tabular}{lccc}
\hline & \multicolumn{3}{c}{ Mortalidad total } \\
\hline Mortalidad de recepción temprana & Semanas con alertas & Semanas sin alertas & Total \\
\hline Semanas con alertas & 15 & 14 & 29 \\
Semanas sin alertas & 4 & 227 & 231 \\
Total & 19 & 241 & $\mathbf{2 6 0}$ \\
\hline
\end{tabular}

*El índice Kappa obtenido para esta tabla es de 0,589 (p=0,000), con un intervalo de confianza al 95\% de (0,418-0,759). 


\section{Correspondencia de las alertas} por exceso de mortalidad, gripe $y$ temperaturas ambientales

En la tabla 4 se resume el número de alertas coincidentes con la temporada de gripe y con altas temperaturas ambientales de MRT y MT.

Tabla 4. Número de alertas por exceso de mortalidad coincidentes con temporadas de gripe y altas temperaturas ambientales según la fuente de mortalidad de recepción temprana (MRT) y de mortalidad total (MT) en el quinquenio 2003-2007.

\begin{tabular}{ccccccc}
\hline & $\mathbf{2 0 0 3}$ & $\mathbf{2 0 0 4}$ & $\mathbf{2 0 0 5}$ & $\mathbf{2 0 0 6}$ & $\mathbf{2 0 0 7}$ & Total \\
\hline Mortalidad de recepción temprana & & & & & & \\
\hline Coincidentes con la gripe & 4 & 2 & 4 & 0 & 3 & $\mathbf{1 3}$ \\
Coincidentes con la calor & 5 & 0 & 4 & 0 & 1 & $\mathbf{1 0}$ \\
Otras causas justificables & 1 & 1 & 3 & 0 & 1 & $\mathbf{6}$ \\
Número total de alertas & $\mathbf{1 0}$ & $\mathbf{3}$ & $\mathbf{1 1}$ & $\mathbf{0}$ & $\mathbf{5}$ & $\mathbf{2 9}$ \\
\hline Mortalidad total & & & & & & \\
\hline Coincidentes con la gripe & 4 & 0 & 4 & 0 & 4 & $\mathbf{1 2}$ \\
Coincidentes con la calor & 5 & 0 & 0 & 0 & 0 & $\mathbf{5}$ \\
Otras causas justificables & 0 & 1 & 0 & 0 & 1 & $\mathbf{2}$ \\
Número total de alertas & $\mathbf{9}$ & $\mathbf{1}$ & $\mathbf{4}$ & $\mathbf{0}$ & $\mathbf{5}$ & $\mathbf{1 9}$ \\
\hline
\end{tabular}

En la figura 2 las curvas de amarillo representan la evolución de la incidencia de gripe para cada año y las barras de color azul las alertas por exceso de mortalidad. 
Mortalidad de recepción temprana (MRT)
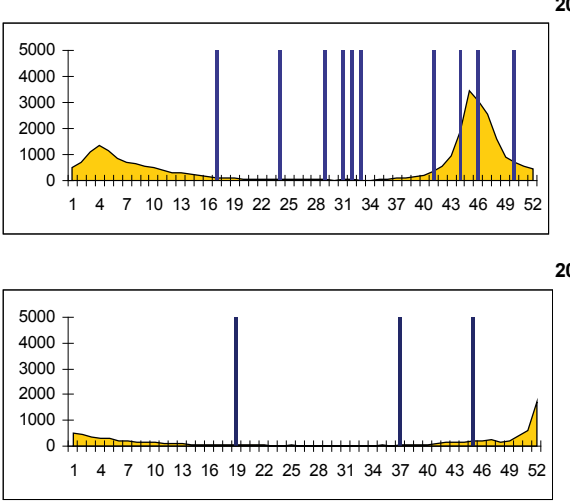

2005
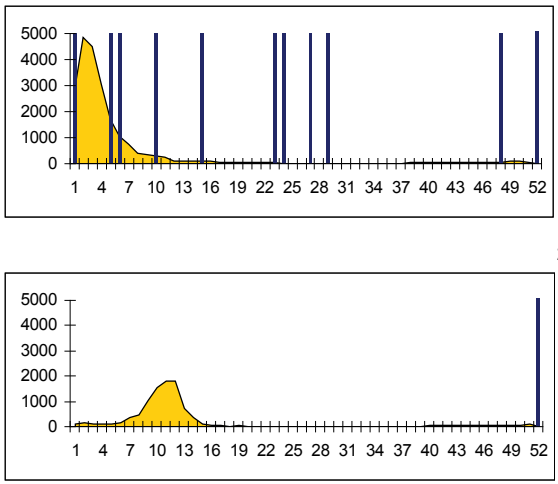

2006

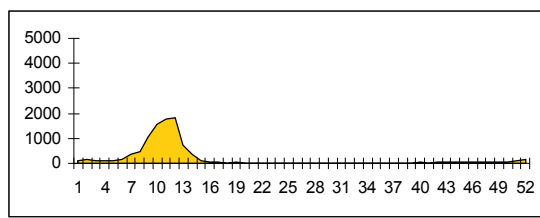

2007
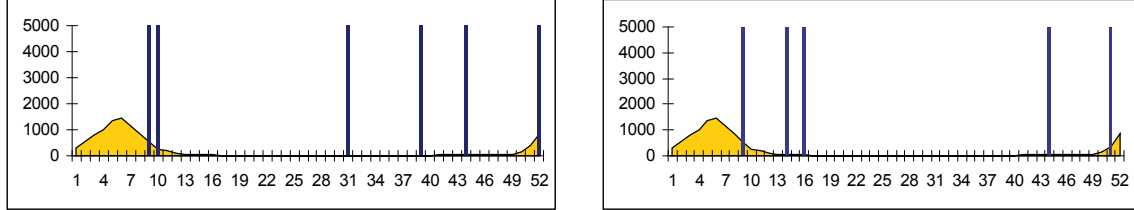

2003

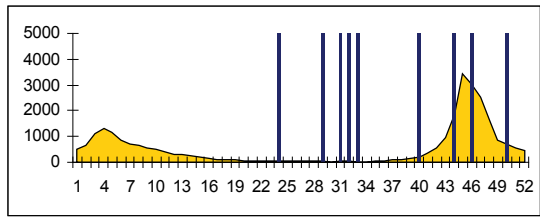

004
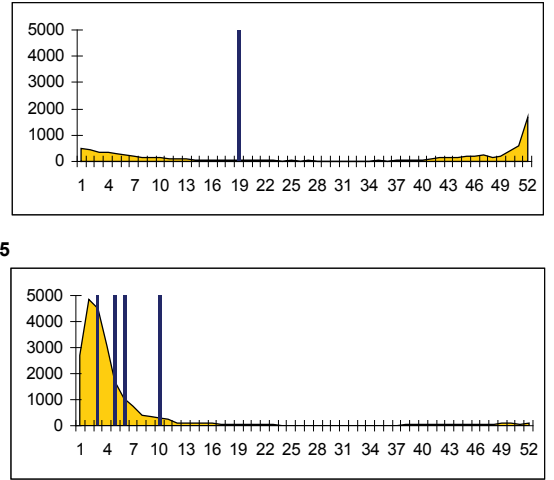

6452

동 de Gripe

- Alertas por exceso de mortalidad

Figura 2 Alertas por exceso de mortalidad y gripe. Se representan de los excesos de mortalidad generadas por la fuente de mortalidad de recepción temprana (columna izquierda) y mortalidad total (columna derecha) junto con los casos de gripe semanales.

En relación a las temperaturas ambientales (Fig. 3) las áreas de azul oscuro representan la media de los máximos y las áreas de color azul claro la media de los mínimos. Las barras de color azul son las alertas por exceso de mortalidad. 


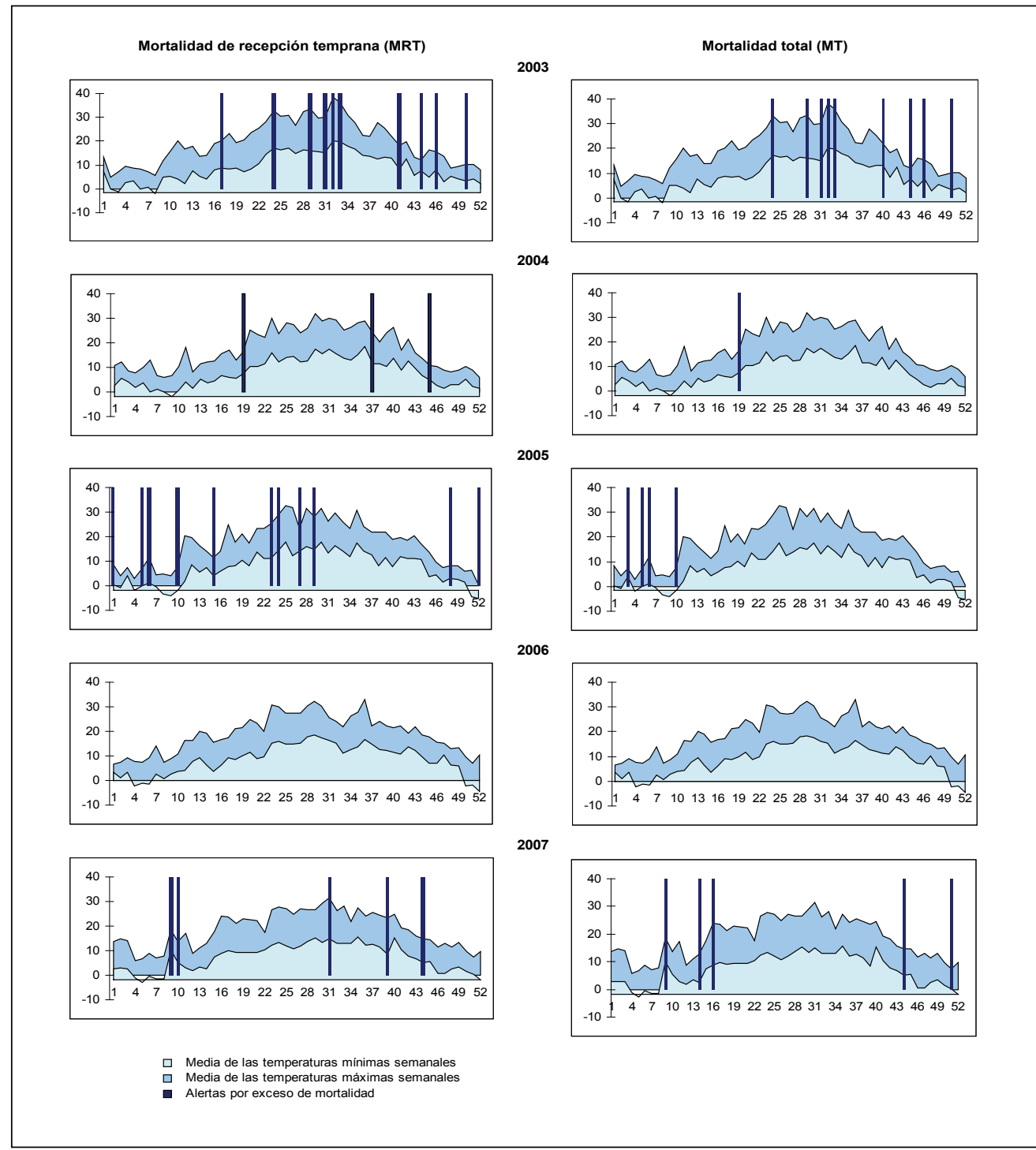

Figura 3. Alertas por exceso de mortalidad y temperatura ambiental. Se representan de los excesos de mortalidad generadas por la fuente de mortalidad de recepción temprana (columna izquierda) y mortalidad total (columna derecha) junto con los históricos de temperatura ambiental.

MRT genera $13(44,5 \%)$ alertas en temporada de gripe. 4, 2, 4, y 3 en 2003, 2004, 2005 y 2007 respectivamente. En la figura 2 , columna de la izquierda se observa un patrón general para las alertas por exceso de mortalidad y gripe ya que las alertas se observan entorno a las ondas de circula- ción del virus gripal. En la onda de 20042005 se observan alertas posteriores que de forma dudosa pueden ser atribuidos a la gripe. Llama la atención la ausencia de alertas en 2006.

En estos cinco años, 10 alertas (34,5\%) se generan en épocas de calor: 5,4 , y 1 en 
2003, 2005 y 2007 respectivamente. Las representaciones de las alertas y las temperaturas ambientales se muestran en la figura 3, columna de la izquierda. El 20,7\% de las alertas de MRT no coincidieron con momentos de actividad gripal ni con temperaturas ambientales extremas.

MT generó 12 (65,1\%) de las alertas coincidiendo con ondas gripales, una menos que MRT (Tabla 4). En la figura 2, columna de la derecha, se observa que estas alertas se distribuyeron en distintos momentos de las ondas de gripe estacional. Se detectaron 5 excesos de mortalidad (26,3\%) coincidiendo con momentos con temperaturas ambientales elevadas. Todos durante la ola de calor de 2003 y además coincidentes con los detectados por la MRT. En ningún otro año se generan alertas en épocas de calor. El 10,5\% de las alertas generadas por la MT no se justifican por alguna de estas dos causas.

\section{DISCUSIÓN}

Analizar la relación de las alertas generadas por las fuentes de MRT y MT frente a la circulación del virus de la gripe y temperaturas ambientales permite conocer mejor el efecto de estos problemas de salud pública sobre la mortalidad. Además, ponen de manifiesto la necesidad de disponer de fuentes de información que permitan la recolección sistemática y continua de la mortalidad para su monitorización precoz, con el fin de contribuir a la toma de decisiones sobre medidas preventivas y planificación de servicios ${ }^{11-13}$.

Los resultados del estudio de las fuentes de MRT y MT en relación a las alertas por exceso de mortalidad revelan desde un punto de vista global una concordancia moderada.

Por otro lado, el análisis enfocado en el estudio de las alertas sobre los históricos de gripe o temperaturas ambientales pone en evidencia aspectos que son deseables para una herramienta de vigilancia. En relación a la gripe, ambas fuentes de datos coinciden en generar varias alertas coincidiendo con las ondas estacionales de gri- pe. En la temporada 2004-2005 se produjo la mayor concentración de alertas en torno a la onda gripal, lo que puede atribuirse a que dicha onda presentó mayor magnitud y su repercusión en la mortalidad ya fue detectada en trabajos previos ${ }^{14}$. El número de alertas coincidiendo con la circulación del virus de la gripe varió ampliamente de unos años a otros, lo que puede deberse a que el virus circulante y la adecuación de la vacuna a los virus circulantes cambian de unas temporadas a otras. También resulta lógico que los excesos de mortalidad tiendan a aparecer preferiblemente en el descenso de las ondas de gripe, ya que la mortalidad puede producirse con retraso como complicación de la gripe ${ }^{15}$. En el otro extremo, en 2006 ninguna de las dos fuentes de información generan alertas por exceso de mortalidad. La actividad gripal de esta temporada tuvo un nivel bajo de intensidad en comparación con años anteriores. Tan solo en la temporada 2000-2001 se declaró un número menor de $\operatorname{casos}^{7}$.

Cuando el estudio se centra en la evaluación de las alertas generadas en temporadas de calor pone de manifiesto un mayor número de alertas observadas con la mortalidad de recepción temprana frente a la mortalidad total. Es decir, mientras que MT solo genera alertas en 2003 (año en el que se registró una ola de calor), MRT muestra además alertas en los años 2005 y 2007. En estos años, aunque no se declararan olas de calor, se observaron varios días consecutivos de altas temperaturas. Estudiar la relación entre temperaturas y mortalidad es un campo de investigación complicado ya que existe la posibilidad de que en ella influyan también otros factores meteorológicos como la humedad o el viento ${ }^{1,16}$.

En relación a las alertas no justificadas por alguna de estas dos causas (el 20,7\% para MRT y 10,5\% para MT) es posible hallar algunas coincidencias. Sin que podamos establecer una relación causal, observamos que el exceso de mortalidad observado en la semana 17 con MRT coincide con la semana posterior a la semana santa de 2003. Por otro lado, la alerta generada 
en la semana 52 del año 2005 (también por MRT) corresponde al periodo navideño.

En este análisis se ha considerado un desglose semanal de las alertas, por lo que no se descarta la posibilidad de que sean alertas únicas continuadas como por ejemplo las tres semanas de exceso identificadas en el verano del 2003. No hay que descartar la posibilidad de que un número de alertas generadas por estos métodos estadísticos sean efecto del azar.

La evaluación de todos los atributos de un sistema de vigilancia puede ayudar a identificar virtudes y defectos de ese sistema. En muchas ocasiones, los esfuerzos se centran en estudiar solo los atributos cuantitativos de los sistemas cuando en realidad también deberían evaluarse otros aspectos cualitativos como pueden ser la sencillez, la flexibilidad y la aceptabilidad. En muchas ocasiones la evaluación correcta de las características cualitativas requiere no solo conocer los aspectos técnicos del sistema sino la opinión de todos sus integrantes y usuarios ${ }^{17}$. Una de las principales ventajes del uso de MRT como fuente de información es la rapidez con la que los datos pueden estar actualizados y disponibles para su explotación. A esto hay que añadir la sencillez de la metodología empleada para su puesta en marcha, por lo que no es necesaria la presencia de personal especializado para llevarla a cabo.

Como características de las fuentes se pueden destacar las siguientes. Dado que la MT recoge todos los fallecidos en Navarra, es una fuente que presenta más potencia y por lo tanto más estabilidad en comparación con la MRT. Esto a su vez puede diluir o suavizar excesos de mortalidad circunscritos a zonas más pequeñas y, por tanto, genera un menor número de alertas. En caso contrario, la MRT se muestra más influenciada por los acontecimientos observados en los 5 registros civiles y, por lo tanto, puede generar un mayor número de alertas. El uso de estas fuentes de información como herramientas para la vigilancia ha de ser complementaria. Mientras que la información de la MRT, una vez contrastada, puede ser utilizada para la actuación temprana, la fuente de MT puede proporcionar una visión retrospectiva más global de lo sucedido. Por otro lado, la informatización de todos los registros civiles permitiría disponer del número total de fallecidos (MT) en un tiempo menor.

Una de las ventajas de esta metodología de los umbrales es que permite evaluar y/o discutir los valores establecidos para la definición de la temporada de gripe y olas de calor mencionadas anteriormente y así poder reajustarlas. La información de estas fuentes puede beneficiarse de la utilización de diversas metodologías como las basadas en series de Fourier ${ }^{18}$ o en series temporales, para corregir algunas de las limitaciones de los umbrales semanales como la variabilidad y estacionalidad y pueden permitir la puesta en marcha de una vigilancia diaria. El uso de la unidad diaria permitiría llevar a cabo otro tipo de análisis diferente. Otra de las limitaciones del uso de las fuentes es que, a menudo, la mortalidad es un indicador tardío de las causas que llevan a la defunción por lo que es interesante complementarla con otras fuentes sobre morbilidad y factores de riesgo.

En conclusión, la combinación entre la sencillez de la metodología, los pocos recursos utilizados para su puesta en marcha y, dado que un gran porcentaje de alertas parecen coincidir con la circulación del virus de la gripe y las altas temperaturas ambientales (se superen o no los criterios para declarar olas de calor), nos hace pensar que el análisis de las fuentes de mortalidad de recepción temprana y de mortalidad total de Navarra pueden ser utilizada como fuente de información para la vigilancia epidemiológica.

\section{Agradecimientos}

Agradecimientos a Fernando Simón, a Inmaculada León y a su equipo del Centro Nacional de Epidemiología por su asesoría. A los registros civiles informatizados de Pamplona, Tudela, Tafalla, Estella y Aoiz por facilitarnos las fuentes de información de mortalidad. Y a los profesionales que están implicados en la recogida de datos para la vigilancia epidemiológica. 


\section{BIBLIOGRAFÍA}

1. Ballester Díez, F. Meteorología y salud. La relación entre la temperatura ambiental y la mortalidad. Rev Esp Salud Pública 1996; 70: 251-259.

2. UNEP DEWA/GRID-Europe, Impacts of summer 2003 heat wave in Europe, 2004.

3. LENGLET A, Simón-Soria F. Exceso de mortalidad en España durante el invierno de 2004-2005. Boletín epidemiológico semanal 2005; 13: 193-195.

4. Martínez Navarro F, Simón-Soria F, López-Abente G. Valoración del impacto de la ola de calor del verano de 2003 sobre la mortalidad. Gac Sanit 2004; 18: 250-258.

5. Ballester F, Corella D, Pérez-Hoyos S, Sáez M, HERVÁs A. Mortality as a function of temperature. A study in Valencia, Spain, 1991-1993. Int J Epidemiol 1997; 26: 551-561.

6. Instituto de Salud Pública de Navarra, Plan de prevención de los efectos de la ola de calor sobre la salud. Navarra 2009. Disponible en: http://www.cfnavarra.es/isp/

7. Instituto de Salud Pública de Navarra, balance de la temporada de gripe 2005-2006 en Navarra. Disponible en: http://www.cfnavarra. es/ISP/documentacion/boletin.htm

8. Web de meteorología y climatología de Navarra, estación de Noáin: http://meteo.navarra. es/estaciones/estacion.cfm?IDEstacion $=16$

9. Josseran L, Nicolau J, Callek̇re N, Astagneau P, BRÜCKER G. Syndromic surveillance based on emergency department activity and crude mortality: two examples. Euro Surveill 2006; 11: pii=668. Disponible en: http://www.eurosurveillance.org/ViewArticle.aspx?ArticleId=668

10. Delgado M, Llorca J, Doménech JM. Estudios para pruebas diagnósticas y factores pronósticos. $3^{\mathrm{a}}$ ed. Barcelona: Signo; 2009.
11. Herrera D, Simón F, Venanzi MJ. Nuevos retos en salud pública: la vigilancia sindrómica como una nueva forma de vigilancia epidemiológica. Gac Sanit 2006; 20: 78-79.

12. Ramírez Fernández R, Ordóñez Iriarte JM. Vigilancia en salud pública: más allá de las enfermedades transmisibles. Gac Sanit 2005; 19: 181-183.

13. Centers for Disease Control and Prevention. Updated guidelines for evaluating public health surveillance systems: recommendations from the guidelines working group. MMWR 2001; 50 (No.RR-13).

14. Brotes de gripe en población vacunada de residencias geriátricas en la temporada 2004-2005. Castilla J y Red de Médicos Centinela para la Vigilancia de la Gripe en Navarra. Med Clin 2006; 126: 35-36.

15. Instituto de Salud Pública de Navarra, Red de Médicos centinela para la vigilancia de la gripe en Navarra. Temporada 2007-2008. Semana 20. Disponible en: http://www.cfnavarra.es/isp/

16. Pajares S, Díaz J, Montero JC, Alberdi JC, MiRÓN IJ. Mortalidad diaria en la Comunidad de Madrid durante el periodo 1986-1991 para el grupo de 45 años a 65 años su relación con la temperatura del aire. Rev Esp Salud Pública 1997; 71: 149-160.

17. De Mateo S, Regidor E. Sistemas de vigilancia de la salud pública: no pidamos peras al olmo. Gac Sanit 2003; 17: 327-331.

18. Alberdi JC, Ordovás M, Quintana F. Construcción y evaluación de un sistema de detección rápida de mortalidad mediante análisis de Fourier. Estudio de un valor con desviación máxima. Rev Esp Salud Pública 1995; 69: 207-217. 